\title{
Avaliação do peso corporal em pacientes com doença de Graves durante o tratamento com metimazol
}

\author{
Evaluation of body weight in patients with Graves' \\ disease during the treatment with methimazole
}

Renata Loureiro Moretto', Ana Beatriz P. Pedro', Alex Carvalho Leite', João Hamilton Romaldini'

${ }^{1}$ Instituto de Assistência Médica ao Servidor Público do Estado de São Paulo (IAMSPE), São Paulo, SP, Brasil
Correspondência para: Renata Loureiro Moretto Rua Desembargador Augusto Botelho, 209, ap. 801 29101-110 - Vila Velha, ES, Brasil rlmoretto@yahoo.com.br

Recebido em 30/Jan/2012 Aceito em 27/Jul/2012

\begin{abstract}
RESUMO
Objetivo: Avaliar a alteração de peso durante o tratamento do hipertiroidismo e correlacioná-la com IL-6 e TNF-alfa. Sujeitos e métodos: Quarenta e dois pacientes foram incluídos. Peso corporal (PC), índice de massa corpórea (IMC), características clínicas e laboratoriais foram registrados. IL-6 eTNF-alfa foram determinados antes do tratamento com metimazol (MMI) e no estado de eutiroidismo. Resultados: O PC foi de $59,62 \pm 11,5 \mathrm{~kg}$ no estado de hipertiroidismo e de $69,91 \pm 14,4 \mathrm{~kg}$ no estado de eutiroidismo $(\mathrm{p}<0,001)$. O IMC aumentou de $23,1 \pm 3,8 \mathrm{~kg} / \mathrm{m}^{2}$ para $27 \pm 4,7 \mathrm{~kg} / \mathrm{m}^{2}$ durante o tratamento $(\mathrm{p}<0,0001)$. Antes da terapia, 66,6\% tinham IMC $<25$ $\mathrm{kg} / \mathrm{m}^{2}$ e $33,3 \%, I M C \geq 25 \mathrm{~kg} / \mathrm{m}^{2}$. No estado de eutiroidismo, $38 \%$ dos pacientes apresentavam $\mathrm{IMC}<25 \mathrm{~kg} / \mathrm{m}^{2}$ e $62 \%, \mathrm{IMC} \geq 25 \mathrm{~kg} / \mathrm{m}^{2}(\mathrm{p}=0,01)$. No estado de eutiroidismo, encontrou-se significativa diminuição nos valores de IL-6 e TNF-alfa, mas nenhuma correlação entre IL-6 eTNF-alfa com PC ou IMC. Conclusão: Um importante aumento no PC e IMC foi observado durante o tratamento do hipertiroidismo e alterações de IL-6 e TNF-alfa relacionam-se somente com o retorno ao eutiroidismo. Arq Bras Endocrinol Metab. 2012;56(6):364-9
\end{abstract}

\section{Descritores}

Hipertiroidismo; fator de necrose tumoral alfa; interleucina-6; metimazol; hormônio tiroidiano; peso corporal

\begin{abstract}
Objective: To evaluate weight change during hyperthyroidism treatment, and to correlate it with IL-6 and TNF-alpha concentrations. Subjects and methods: Forty two patients were included. Body weight (BW), body mass index (BMI), clinical and laboratory characteristics were recorded. IL-6 andTNF-alpha were determined before treatment with methimazole (MMI) and in euthyroidism. Results: BW was $59.62 \pm 11.5 \mathrm{~kg}$ in hyperthyroidism, and $69.91 \pm 14.4 \mathrm{~kg}$ in euthyroidism ( $<0.001$ ). BMI increased from $23.1 \pm 3.8 \mathrm{~kg} / \mathrm{m}^{2}$ to $27 \mathrm{~kg} / \mathrm{m}^{2} \pm 4.7$ during treatment ( $<0.0001$ ). Before treatment, $66.6 \%$ subjects had $\mathrm{BMI}<25 \mathrm{~kg} / \mathrm{m}^{2}$ and $33.3 \%, \mathrm{BMI} \geq 25 \mathrm{~kg} / \mathrm{m}^{2}$. In euthyroidism, $38 \%$ of patients had $\mathrm{BMI}<25 \mathrm{~kg} / \mathrm{m}^{2}$ and $62 \%, \mathrm{BMI} \geq 25 \mathrm{~kg} / \mathrm{m}^{2}(\mathrm{p}=0.01)$. In euthyroidism, we found a significant reduction in IL-6 and TNF-alpha concentrations, but no correlation between IL-6 andTNF-alpha, and BW or BMI. Conclusion: An important increase in BW and BMI was observed during hyperthyroidism treatment, and IL-6 and TNF-alpha alterations were only related with return to euthyroidism. Arq Bras Endocrinol Metab. 2012;56(6):364-9
\end{abstract}

Keywords

Hyperthyroidism; tumour necrosis factor alpha; interleukin-6; methimazole; thyroid hormone; body weight

\section{INTRODUÇÃO}

$\mathrm{O}$ hipertiroidismo tendo como causa a doença de Graves é caracterizado imunologicamente por infiltração linfocitária da glândula tiroide e por ativação do sistema imune com elevação dos linfócitos T circulantes, aparecimento de autoanticorpos que se ligam ao receptor do TSH (TRAb) e estimulam o crescimento e a função glandular (1-4). O hormônio tiroidiano atua 
nos tecidos e órgãos do sistema corpóreo, aumentando a termogênese e a taxa metabólica (1-4).

Uma das complicações do hipertiroidismo não tratado é a perda de peso, associada ao aumento de apetite (5-7). Pacientes portadores de hipertiroidismo normalmente perdem peso mesmo com o aumento de apetite e ingesta calórica e retornam ao peso após o tratamento (811). Alguns pacientes com hipertiroidismo não apresentam perda de peso; aproximadamente $5 \%$ a $10 \%$ ganham-no, refletindo o aumento de apetite e da taxa metabólica decorrente do excesso de hormônio tiroidiano (12).

O hipertiroidismo estimula o apetite por longo período, talvez irreversivelmente, resultando em ganho de peso (13). Entretanto, a prevalência de hipotiroidismo durante o tratamento é de $42 \%$ a $67 \%$ (14), e esses pacientes também estão sujeitos a ganho de peso, do mesmo modo que pacientes com hipotiroidismo primário (15). Além disso, muitos pacientes não retornam ao peso pré-tratamento, mesmo após a reposição com levotiroxina $(8,9,10,13)$.

Distúrbio no sistema imunológico mediado pelo desequilíbrio entre as citocinas Thl e Th2 pode ser a causa primária da doença de Graves. A resposta Th2 é prevalente na doença de Graves (16), com aumento de interleucina 2 (IL-2) e de seu receptor (IL-2R), interleucina 6 (IL-6) e de seu receptor interleucina 6R (IL$-6 \mathrm{R})$, interleucina 1 beta (IL-1 beta), fator de necrose tumoral alfa (TNF-alfa) e interleucina 18 (IL-18); essas citocinas se normalizam após a restauração do estado de eutiroidismo durante o tratamento com metimazol (MMI) (17-19). Recentes estudos revelaram que as quimiocinas (em especial a CXCL-10) também se encontram elevadas no hipertiroidismo (20). Portanto, o aumento significativo de citocinas e quimiocinas circulantes e consequente redução de seus valores pós-restauração do eutiroidismo sugerem sua importância na imunidade da doença de Graves e, por consequência, no metabolismo energético $(20,21)$.

Obesidade e inflamação estão associadas com a resistência à insulina e à síndrome metabólica (22-25). Indivíduos obesos têm excesso de tecido adiposo, o qual é metabolicamente ativo, secretando citocinas e adiponectinas capazes de modular reações imunes (22-27).

IL-6 é uma citocina pró-inflamatória secretada por vários tecidos, tais como músculos, leucócitos, hepatócitos, e aproximadamente $10 \%$ do total da IL-6 é produzido exclusivamente pelo tecido adiposo (25). Concentrações séricas de IL-6 estão elevadas em obesos, se comparadas a indivíduos de peso normal, e correla- cionam-se positivamente ao índice de massa corpórea (IMC) (27).

A expressão e a secreção de TNF-alfa encontram-se elevadas no tecido adiposo e a neutralização de sua secreção melhora a resistência à insulina $(25,26)$. Vários estudos sugerem um papel para o TNF-alfa com resistência à insulina encontrada na obesidade (25-27). Desse modo, as concentrações de IL-6 e TNF-alfa encontram-se elevadas no tecido adiposo e no soro dos pacientes obesos (22-28).

Existe uma relação inversa entre os níveis plasmáticos de adiponectina e essas citocinas, sugerindo que IL-6 e TNF-alfa inibam a adiponectina, um importante sensibilizador da insulina (26-28). Também foi demonstrado que o TNF-alfa inibe a produção de leptina nos adipócitos subcutâneo e omental em pacientes com obesidade mórbida (22). Os valores séricos de TNF-alfa correlacionam-se ao IMC, porcentagem de gordura no corpo e hiperinsulinemia $(25,26)$ e, quando ocorre diminuição do peso, seus valores diminuem $(25,26)$.

O objetivo deste estudo foi avaliar a possível alteração de peso corpóreo nos pacientes com hipertiroidismo decorrente da doença de Graves, durante o tratamento com MMI, no estado de eutiroidismo, e correlacioná-la às prováveis variações na concentração sérica de TNF-alfa e IL-6.

\section{SUJEITOS E MÉTODOS}

\section{Pacientes}

Foram estudados 42 consecutivos pacientes portadores de hipertiroidismo com doença de Graves. Destes, 7 $(16,6 \%)$ eram do sexo masculino e $35(83,3 \%)$, do sexo feminino, média de 40,5 $\pm 0,2$ anos (desvio-padrão).

Os critérios de inclusão foram: história clínica, sinais e sintomas de hipertiroidismo sem tratamento prévio, bócio difuso, fração livre de T4 (T4L) e T3 elevados, concentração de hormônio estimulante da tiroide (TSH) suprimida, presença de anticorpo estimulante da tiroide (TRAb) e/ou captação aumentada de iodo radioativo 131 nas $24 \mathrm{~h}$ (24h-RAIU) com distribuição homogênea, caracterizando doença de Graves. Foram excluídos pacientes com doenças associadas à obesidade e pacientes grávidas.

No início, todos os pacientes foram tratados com $30 \mathrm{mg}$ a $40 \mathrm{mg}$ de MMI, ajustando-se a dose gradativamente entre seis e oito semanas, além de manter a avaliação clínica e dosagens séricas de TSH, T4L e T3. 
A dose de manutenção foi de $5 \mathrm{mg}$ a $15 \mathrm{mg}$ de MMI. Todos os pacientes atingiram o estado de eutiroidismo, caracterizado pelo quadro clínico, valores normais de TSH, T4L e T3 (Tabela 1).

Tabela 1. Parâmetros clínicos, bioquímicos e imunológicos no diagnóstico e após a normalização da função tiroidiana durante 0 tratamento com metimazol

\begin{tabular}{lccc}
\hline & Hipertiroidismo & Eutiroidismo & P \\
\hline TSH (mU/L) & $0,009 \pm 0,001$ & $1,04 \pm 1,13$ & $<0,0001$ \\
T4L (pmol/L) & $71,4 \pm 25,2$ & $11,2 \pm 6,5$ & $<0,0001$ \\
T3 (nmol/L) & $6,8 \pm 2,4$ & $2,9 \pm 1,5$ & $<0,0001$ \\
TPOAb (mU/L) & $509,26 \pm 951,67$ & $340,42 \pm 797,39$ & 0,38 \\
TGAb (mU/L) & $210,17 \pm 486,11$ & $74,95 \pm 160,23$ & 0,09 \\
TRAb (IU/L) & $67,9 \pm 69,75$ & $65,47 \pm 81,91$ & 0,66 \\
\hline
\end{tabular}

Valores representam média e 2 desvios-padrão.

As concentrações séricas de IL-6 e TNF-alfa foram medidas antes do tratamento e no estado de eutiroidismo durante o tratamento com MMI.

Peso corporal e IMC foram avaliados no início do tratamento na fase de hipertiroidismo e no estado de eutiroidismo.

\section{MÉTODOS}

As concentrações séricas de TSH, T4L e T3 foram determinadas a partir do ensaio de quimioluminométrico (Diagnostic Products Company, Los Angeles, CA, USA) com valores de referência em nosso laboratório de $0,3 \mathrm{mU} / \mathrm{L}$ a $4,5 \mathrm{mU} / \mathrm{L}$ para o TSH, $1,2 \mathrm{nmol} / \mathrm{L}$ a $3,1 \mathrm{nmol} / \mathrm{L}$ para T3 e de $8,8 \mathrm{pmol} / \mathrm{L}$ a $23,2 \mathrm{pmol} / \mathrm{L}$ para T4L, respectivamente. As concentrações de TRAb foram determinadas por ensaio radiorreceptor (R.S.R. Limited, Cardiff, Wales, UK), cujos valores-controles foram menores que $11 \mathrm{UI} / \mathrm{mL}$. As concentrações de $\mathrm{TgAb}$ e TPOAb foram determinadas por ensaio radiorreceptor (R.S.R. Limited, Cardiff, Wales, UK), cujos valores-controles foram menores que $1 \mathrm{UI} / \mathrm{mL}$.

Os ensaios séricos das citocinas foram coletados em jejum, processados em 30 minutos e estocados a $-70^{\circ} \mathrm{C}$. A determinação da concentração sérica de cada citocina foi feita em único ensaio para aumentar a precisão do método. IL-6 e TNF-alfa foram avaliadas pelo ensaio imunoenzimático que utiliza proteínas recombinantes (ELISA) (Quantikine, R\&D Systems, Minneapolis, MN). O limite de detecção foi de $0,7 \mathrm{pg} / \mathrm{mL}$ para IL-6 e 4,4 pg/mL para TNF-alfa. Os coeficientes de variação para IL-6 e TNF-alfa foram 3,1\% e 5,2\%, respectivamente. A variação diária foi menor que $7 \%$ para todos os ensaios.

A análise estatística foi realizada pelo software SAS Statistical (Statistical Analysis System, version 8.1, Cary, NC, USA, 1999-2000).

A comparação entre os pacientes portadores de hipertiroidismo antes do tratamento com MMI e após a normalização da função tiroidiana foi realizada pelo método Wilcoxon rank-sum test. O teste de Mann-Whitney foi usado para comparar os dados dos diferentes grupos. O método de Fischer's exact test foi usado para associação entre os dois fatores explícitos. A relação entre cada citocina e os hormônios tiroidianos foi calculada pelo método de correlação de Spearman. O nível de significância usado foi de $\mathrm{p}<0,05$.

Este estudo foi aprovado pela Comissão de Ética em Pesquisa do Hospital do Servidor Público do Estado de São Paulo.

\section{RESULTADOS}

Os pacientes com hipertiroidismo necessitaram de um período médio de $10,3 \pm 6,3$ semanas para atingirem o eutiroidismo com tratamento com MMI (Tabela 1). A média de duração da terapia foi de $15,1 \pm 2,6$ meses (13-23 meses).

A variação do peso corporal foi de $59,62 \mathrm{~kg} \pm 11,5$ $\mathrm{kg}$ para $69,91 \mathrm{~kg} \pm 14,4 \mathrm{~kg}(\mathrm{p}<0,001)$ na mudança do estado de hipertiroidismo para eutiroidismo (Figura l), enquanto o IMC (Figura 2) aumentou de $23,1 \mathrm{~kg} / \mathrm{m}^{2}$ $\pm 3,8 \mathrm{~kg} / \mathrm{m}^{2}$ para $27 \mathrm{~kg} / \mathrm{m}^{2} \pm 4,7 \mathrm{~kg} / \mathrm{m}^{2}(\mathrm{p}<0,0001)$. A média de ganho de peso foi semelhante no sexo feminino e no masculino.

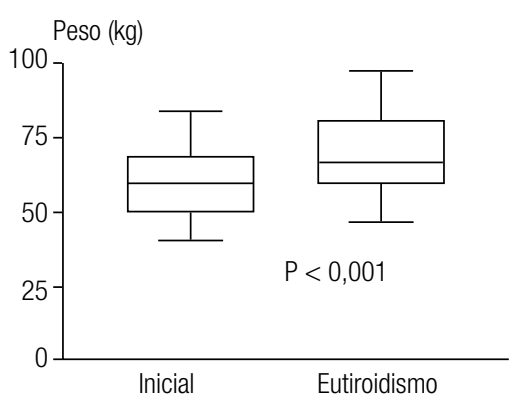

Figura 1. Gráfico Box e Whisker do peso corpóreo de pacientes com hipertiroidismo, antes do tratamento (inicial) e no eutiroidismo. A linha da caixa (boxe) indica 0 valor de mediana dos dados. A caixa contém a metade $(50 \%)$ dos dados. 0 limite superior da caixa indica o percentil $75 \%$ dos dados e o limite inferior da caixa indica o percentil de $25 \%$. 


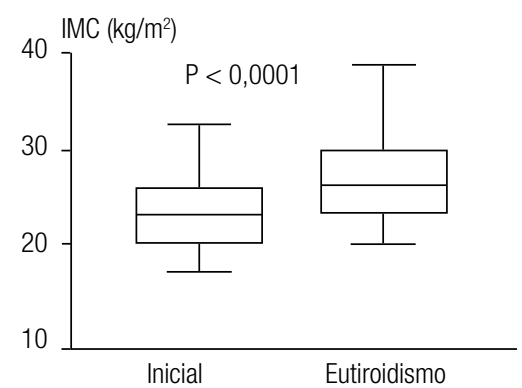

Figura 2. Gráfico Box e Whisker do índice de massa corpórea (IMC) dos pacientes com hipertiroidismo antes do tratamento (inicial) e no eutiroidismo. A linha da caixa (boxe) indica o valor de mediana dos dados. A caixa contém a metade $(50 \%)$ dos dados. 0 limite superior da caixa indica o percentil $75 \%$ dos dados e 0 limite inferior da caixa indica 0 percentil de $25 \%$.

Antes do tratamento, 66,6\% $(\mathrm{n}=28 / 42)$ dos pacientes tinham IMC menor a $25 \mathrm{~kg} / \mathrm{m}^{2}$ e $33,3 \%(\mathrm{n}=$ $14 / 42$ ), IMC maior ou igual a $25 \mathrm{~kg} / \mathrm{m}^{2}$. No estado de eutiroidismo, 38\% ( $\mathrm{N}=16 / 42)$ dos pacientes apresentavam IMC menor a $25 \mathrm{~kg} / \mathrm{m}^{2}$ e $62 \%(\mathrm{~N}=26 / 42)$, IMC maior ou igual a $25 \mathrm{~kg} / \mathrm{m}^{2}(\mathrm{p}=0,01)$ (Figura 3 ).

As mudanças percentuais de peso e IMC foram, respectivamente, $17,2 \% \pm 11,3 \%$ e $18,0 \% \pm 11,9 \%(\mathrm{p}<$ $0,001)$. No estado de eutiroidismo, 37 pacientes $(88 \%)$ ganharam mais de $5 \%$ de peso em relação ao início (p $=0,0001)$.

Antes do tratamento com MMI, os valores séricos TNF-alfa encontrados nos pacientes com hipertiroidismo foram de $33,32 \mathrm{pg} / \mathrm{mL} \pm 69,86 \mathrm{pg} / \mathrm{mL}$ e, após a restauração do eutiroidismo, foi observado um declínio significativo para $28,8 \mathrm{pg} / \mathrm{mL} \pm 84,35 \mathrm{pg} / \mathrm{mL}(\mathrm{p}<$ 0,02 ) (Figura 4). Os valores séricos de IL-6 também diminuíram significativamente $(\mathrm{p}<0,03)$ no eutiroidis$\mathrm{mo}$, de $42,9 \mathrm{pg} / \mathrm{mL} \pm 191,47 \mathrm{pg} / \mathrm{mL}$ para $37,85 \mathrm{pg} /$ $\mathrm{mL} \pm 189,82 \mathrm{pg} / \mathrm{mL}$ (Figura 5).

Não foram observadas correlações significativas, antes do tratamento, entre os valores séricos de TNF-alfa e peso corporal $(\mathrm{r}=-1,1 ; \mathrm{p}=0,47)$ ou IMC $(\mathrm{r}=-0,1 ; \mathrm{p}$ $=0,9)$ ou, no estado de eutiroidismo (peso corporal, $\mathrm{r}=$ $-0,4 ; \mathrm{p}=0,74$ e IMC, $\mathrm{r}=-0,01 ; \mathrm{p}=0,91)$. Do mesmo modo, as correlações dos valores séricos de IL-6, antes do tratamento, com o peso corporal $(\mathrm{r}=0,23 ; \mathrm{p}=0,12)$ ou IMC $(r=-0,22 ; p=0,11)$ e, no estado de eutiroidismo (peso corporal, $r=0,06 ; p=0,62$ e IMC, $r=0,05$; $\mathrm{p}=0,54)$, não foram significativas. Nenhuma relação também pôde ser encontrada entre peso corporal e IMC e valores séricos de TRAb, TPOAb, TgAb, T4L, T3 e TSH antes do tratamento e no estado de eutiroidismo.

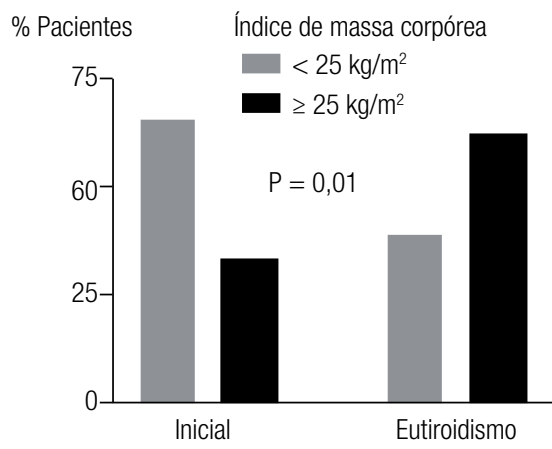

Figura 3. Porcentagem de pacientes de acordo com índice de massa corpórea (IMC) antes do tratamento com metimazol (MMI) e no estado de eutiroidismo.

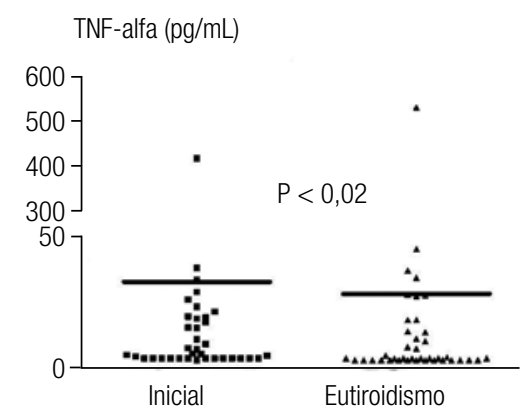

Figura 4. Concentração sérica de TNF-alfa de pacientes hipertiróideos antes do tratamento (inicial) e no eutireoidismo. Linha corresponde à média dos valores.

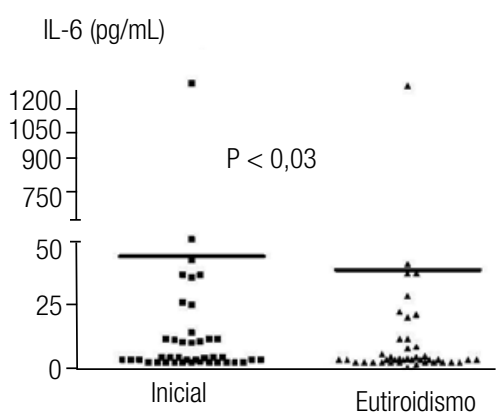

Figura 5. Concentração sérica de IL-6 de pacientes hipertiróideos antes do tratamento (inicial) e no eutiroidismo. Linha corresponde à média dos valores.

\section{DISCUSSÃO}

Em pacientes com hipertiroidismo, a perda de peso se deve à redução de tecidos adiposo, muscular e de massa óssea $(7,29)$. A redução de peso associada com o hipertiroidismo é de aproximadamente 15\% (7); entretanto, $5 \%$ a $10 \%$ dos pacientes podem ganhar peso, o que 
reflete o excesso de apetite consequente do aumento do metabolismo pelo excesso de hormônio tiroidiano $(10,12)$.

A mudança da composição corpórea durante o tratamento de hipertiroidismo é motivo de controvérsia. Alguns trabalhos mostram aumento de peso, IMC, massa magra e massa gorda em paralelo ao tratamento $(9,10)$. Embora ganho de peso refletindo predominantemente ganho de massa muscular e óssea tenha sido descrito (11), finalmente se pode observar ganho de massa muscular e tecido adiposo, mas não de massa óssea (30).

O tratamento da doença de Graves com drogas antitiroidianas pode gerar aumento de peso entre 2,5\% e $16 \%$ do peso antes do tratamento (9). Além disso, a prevalência de hipotiroidismo após o tratamento varia de $42 \%$ a $67 \%(14,15)$ e esses pacientes têm a mesma probabilidade de aumento de peso que aqueles com hipotiroidismo primário. Cerca de $80 \%$ dos pacientes com hipotiroidismo pós-tratamento da doença de Graves ganham peso, apesar da reposição com levotiroxina $(7,15,31)$. No entanto, anos depois da suspensão das drogas antitiroidianas, os pacientes em remissão têm $11 \%$ de risco de desenvolver hipotiroidismo espontâneo (32). Esse ganho de peso durante e depois do tratamento pode ser decorrente da discrepância que se instala entre apetite e mudança do metabolismo do estado de tirotoxicose para o eutiroidismo, seguindo a normalização das concentrações dos hormônios tiroidianos. Além disso, o metabolismo diminui em relação à fase de tirotoxicose decorrente do ganho de massa magra corporal, a qual sofre grande perda durante o hipertiroidismo ativo. Entretanto, foi demonstrado que o aumento da ingesta calórica no hipertiroidismo pode reaparecer ou continuar durante o tratamento, levando o paciente a um significativo aumento de peso (8-10).

Jansson e cols. (9) sugerem que, após o hipertiroidismo, ocorre um distúrbio na regulação neuroquímica do apetite e da regulação do peso corporal, de modo que o controle central do apetite não sinaliza a redução do metabolismo durante o tratamento do hipertiroidismo. Segundo Ortega e cols. (33), valores baixos de T3 livre e não os valores de T4 livre agem como fator independente para oxidação lipídica, padrão metabólico e mudança de peso corporal em pacientes com eutiroidismo. Gul e cols. demonstraram que pequenas reduções nos valores de T4 livre (ainda no intervalo de normalidade) estão relacionadas com o ganho de peso e pequenas alterações na função tiroidiana estão associadas com o IMC (34), enquanto pequena elevação dos valores de
TSH no soro (hipotiroidismo subclínico) pode influenciar na prevalência de obesidade da população (35).

No presente estudo, observamos aumento de peso durante o tratamento de hipertiroidismo com MMI. Do mesmo modo que Abid e cols. (8), $88 \%$ dos pacientes aumentaram mais de $5 \%$ do peso corporal inicial, durante tratamento com MMI. Também observamos que os valores de TNF-alfa diminuíram no estado de eutiroidismo, apesar do ganho de peso. Essa situação pode parecer paradoxal, mas, como a caquexia e a obesidade são condições inflamatórias, consequentemente esses mediadores podem estar envolvidos em ambos os processos (23). Os valores de IL-6 diminuíram durante o tratamento com MMI. A diminuição na concentração sérica dessas citocinas ocorreu por provável instalação do estado de eutiroidismo e pela ação anti-inflamatória e imunomoduladora do MMI $(20,21)$.

Neste estudo, o tamanho da amostra não teve poder suficiente para demonstrar significante relação entre ganho de peso e alterações nas citocinas TNF-alfa e IL-6. A despeito de não podermos demonstrar que essas duas citocinas foram diretamente responsáveis pelo ganho de peso, este estudo amplia nossos conhecimentos sobre a diminuição na concentração dessas citocinas no tratamento do hipertiroidismo com MMI. Futuros trabalhos serão necessários para correlacionar as citocinas ao importante ganho de peso corporal observado no tratamento com MMI do hipertiroidismo causado pela doença de Graves $(36,37)$. O presente estudo sugere que o monitoramento de peso dos pacientes durante a terapia do hipertiroidismo, aconselhando dietas hipocalóricas e a prática de atividade física regular, possa evitar o ganho de peso. Apesar de não ser o objetivo deste estudo, uma das principais causas da falta de aderência ao tratamento clínico do hipertiroidismo (38) pode estar relacionada ao aumento de peso e, restando comprovar que, com a prevenção do ganho de peso, observaremos maior adesão à terapia.

Declaração: os autores declaram não haver conflitos de interesse científico neste estudo.

\section{REFERÊNCIAS}

1. Weetman AP. Graves' disease. N Engl J Med. 2000;343(17):1236-48.

2. Andrade VA, Gross JL, Maia AL. Tratamento do hipertireoidismo da doença de Graves. Arq Bras Endocrinol Metab [online]. 2001;45(6):609-18.

3. Neves C, Alves M, Delgado JL, Medina JL. Doença de Graves. Arquimed. 2008;22(4/5):137-46.

4. Girgis CM, Champion BL, Wall JR. Current concepts in Graves' disease. Ther Adv Endo Metab. 2011;2(3):135-44. 
5. Bahn RS, Burch HB, Cooper DS, Garber JR, Greenlee MC, Klein I, et al. Hyperthyroidism and other causes of thyrotoxicosis: management guidelines of the American Thyroid Association and American Association of Clinical Endocrinologists. Endocr Pract. 2011:17(3):e1-65.

6. Sestoft L. Metabolic aspects of the calorigenic effect of thyroid hormone in mammals. Clin Endocrinol. 1980;13:489-586.

7. Hoogwerf BJ, Nutall FQ. Long-term weight regulation in hyperthyroid and hypothyroid subjects. Am J Med. 1984;76:963-70.

8. Abid M, Billington CJ, Nuttall FQ. Thyroid function and energy intake during weight gain following treatment of hyperthyroidism. J Am Coll Nutr. 1999;18(2):189-93.

9. Jansson S, Berg G, Lindstedt G, Michanek A, Nystrom E. Overwigth-a common problem among women treated for hypothyroidism. Postgrad Med J. 1993;69:107-11.

10. Dale J, Daykin J, Holder R, Sheppard MC, Franklyn JA. Weight gain following treatment of hyperthyroidism. Clin Endocrinol. 2001;55:233-9.

11. De La Rosa RE, Hennessey JV, Tucci JR. A longitudinal study of changes in body mass index and total body composition after radioiodine treatment for thyrotoxicosis. Thyroid. 1997;7:401-5.

12. Gurney C, Hall R, Harper M, Owen SG, Roth M, Smart GA. Newcastle thyrotoxicosis index. Lancet. 1970;2:1275-8.

13. Alton S, O'Malley BP. Dietary intake in thyroxicosis before and after adequate carbimazole therapy; the impact of dietary advice. Clin Endocrinol. 1985;23:517-20.

14. Choo YK, Yoo WS, Kim DW, Chung HK. Hypothyroidism during antithyroid drug treatment with methimazole is a favorable prognostic indicator in patient with Graves' disease. Thyroid. 2010;20(9):949-54.

15. Franklyn JA, Daykin J, Drolc Z, Farmer M, Sheppard MC. Long-term follow-up of treatment of thyrotoxicosis by three different methods. Clin Endocrinol. 1991;34:71-6.

16. Fountoulakis $S$, Tsatsoulis $A$. On the pathogenesis of autoimmune thyroid disease: a unifying hypothesis. Clin Endocrinol (Oxf). 2004;60:397-409.

17. Celik I, Akalin S, Erbas T. Serum level of interleukin 6 and tumor necrosis factor-alpha in hyperthyroid patients before and after propylthiouracil treatment. Eur J Endocrinol. 1995;132:668-72.

18. Díez J, Hernanz A, Medina S, Bayón C, Iglesias P. Serum concentrations of tumour necrosis factor-alpha (TNF-alpha) and soluble TNF-alpha receptor p55 in patients with hypothyroidism and hyperthyroidism before and after normalization of thyroid function. Clin Endocrinol (Oxf). 2002;57:515-21.

19. Salvi M, Girasole G, Pedrazzoni M, Passeri M, Giuliani N, Minelli $\mathrm{R}$, et al. Increased serum concentrations of interleukin-6 (IL-6) and soluble IL-6 receptor in patients with Graves' disease. J Clin Endocrinol Metab. 1996;81(8):2976-9.

20. Leite AC, Pedro, ABP, Romaldini JH. Influence of methimazole and radioactive iodine treatment in the serum levels of the chemokine CXCL10 in hyperthyroid patients with Grave's disease. Horm Met Res. 2011;43:194-9.

21. Pedro ABP, Romaldini $\mathrm{JH}$, Takei $\mathrm{K}$. Changes of serum cytokines in hyperthyroid Graves' disease patients at diagnosis and during methimazole treatment. Neuroimmunomodulation. 2011;18:45-51.
22. Fawcett $R L$, Waechter $A S$, Williams LB, Zhang $P$, Louie $R$, Jones $R$, et al. Tumor necrosis factor alpha inhibits leptin production in subcutaneous and omental adipocytes from morbidly obese humans. J Clin Endocrinol Metab. 2000;85:530-5.

23. Fantuzzi G. Adipose tissue, adipokines, and inflammation. J Allergy Clin Immunol. 2005;115(5):911-9.

24. Bruun JM, Lihn AS, Verdich C, Pedersen SB, Toubro S, Astrup A, et al. Regulation of adiponectin by adipose tissue-derived cytokines: in vivo and in vitro investigations in humans. Am J Physiol Endocrinol Metab. 2003;285:E527-33.

25. Ronti T, Lupattelli G, Mannarino $E$. The endocrine function of adipose tissue: an update. Clin Endocrinol. 2006;64:335-65.

26. Warne JP.Tumor necrosis factor alpha: a key regulator of adipose tissue mass. J Endocrinol. 2003;177:351-5.

27. Carvalho MHC, Colaço AL, Fortes ZB. Citocinas, disfunção endotelial e resistência à insulina. Arq Bras Endocrinol Metab. 2006;50(2):304-12.

28. Fain JN, Bahouth SW, Madan AK. TNF-alpha release by nonfat cells of human adipose tissue. Int $\mathrm{J}$ Obes Relat Metab Disord. 2004;28:616-22.

29. Zimmerman-BelsingT, Dreyer M, Holst JJ, Feldt-Rasmussen U.The relationship between the serum leptin concentrations of thyrotoxic patients during treatment and their total fat mass is different from that of normal subjects. Clin Endocrinol. 1998;49:589-95.

30. Lonn L, Stenlof K, Ottoson M, Lindroos AK, Nystrom E, Sojostrom L. Body weight and body composition changes after treatment of hyperthyroidism. J Clin Endocrinol Metab. 1998;83:4269-73.

31. Child DF, Mughni MA, Hudson P, Williams CP, Harvey JN. Hyperthyroidism and radio-iodine therapy in a district general hospital. J R Soc Med. 1994;87:578-80.

32. De Moraes AVC, Pedro ABP, Romaldini JH. Spontaneous hypothyroidism in the follow up of graves hyperthyroid patients treated with antithyroid drugs. Southern Med J. 2006;99:1068-107.

33. Ortega E, Pannacciulli N, Bogardus C, Krakoff J. Plasma concentrations of free triiodothyronine predict weight change in euthyroid persons. Am J Clin Nutr. 2007;85(2):440-5.

34. Gul OO, Ersoy C, Gul CB, Guclu M, Ibanoglu MS, Cangur S, et al. The relationship between minor alterations in thyroid function in euthyroid patients and obesity. Endocrinologist. 2010;20(6):304-7.

35. Knudsen N, Laurberg P, Rasmussen LB, Bulow I, Perrild H, Ovesen $L$, et al. Small differences in thyroid function may be important for body mass index and the occurrence of obesity in the population. J Clin Endocrinol Metab. 2005;90:4019-24.

36. Hsieh CJ, Wang PW. Serum concentrations of adiponectin in patients with hyperthyroidism before and after control of thyroid function. Endocr J. 2008;55(3):489-94.

37. Charle BA, Doumatey A, Huang H, Zhou J, Chen G, Shriner D, et al. The Roles of IL-6, IL-10, and IL-1RA in obesity and insulin resistance in African-Americans. J Clin Endocrinol Metab. 2011;96:E2018-22.

38. Cooper DS. Antithyroid drugs in the management of patients with Graves' disease: an evidence-based approach to therapeutic controversies. J Clin Endocrinol Metab. 2003;88:3474-81. 